\title{
ESTUDO PROSPECTIVO CONTROLADO RANDOMIZADO. COMPARANDO DUAS FORMAS DE TRATAMENTO PARA O SITIO DOADOR DE ENXERTO ÓSSEO EM CIRURGIA DA COLUNA VERTEBRAL
}

\author{
CONTROLLED RANDOMIZED PROSPECTIVE STUDY COMPARING TWO FORMS OF \\ TREATING THE BONE GRAFT DONOR SITE IN VERTEBRAL-SPINE SURGERY \\ ESTUDIO PROSPECTIVO CONTROLADO RANDOMIZADO. COMPARANDO DOS TIPOS DE \\ TRATAMIENTO PARA EL SITIO DONADOR DEL INJERTO ÓSEO EN CIRURGÍA \\ DE COLUMNA VERTEBRAL
}

luiz Claudio lacerda Rodrigues'i , Adalberto Bortoletto², Marcelo Hide Matsumoto ${ }^{3}$

\begin{abstract}
RESUMO
Objetivo: Comparar a colocação de geléia Gelfoan ${ }^{\circledR}$ versus a colocação apenas de dreno de sucção na área doadora, avaliando a dor pós-operatória, presença de complicações e seguimento dos pacientes. Métodos: Foram randomizados por programa de computador 30 pacientes, sendo que em 14 pacientes foi utilizado Gelfoan ${ }^{\circledR}$ com anestésico e em 16 pacientes utilizamos dreno de sucção. Todos os pacientes foram avaliados em cinco períodos consecutivos, sendo avaliada a dor baseada na Escala Visual Analógica (VAS) e observado a incidência de complicações. Foram incluídos pacientes com abordagem anterior ou posterior, sendo considerado como fonte doadora única a crista ilíaca. Foram incluídos pacientes com 18 a 70 anos de idade de ambos os sexos. Resultados: Foi observado que a dor pós-operatória foi menor com Gelfoan ${ }^{\circledR}$ nas primeiras avaliações, mas na média o resultado foi semelhante, não apresentando diferença estatística entre ambas as técnicas. Na média das avaliações, a escala visual analógica de dor foi de dois; não se observando complicações na área da retirada do enxerto. Conclusão: A abordagem com Gelfoan ${ }^{\circledR} \mathrm{com}$ bupivacaina melhora a dor nas primeiras $48 \mathrm{~h}$ pós-cirurgia, quando comparada com dreno de sucção a vácuo, mas na média ambas a técnicas são seguras, com presença de dor residual nas duas intervenções, mas com baixo índice de complicações. Não observamos complicações em nosso estudo.
\end{abstract}

Descritores: Prospectivo; Controlado, Enxerto ósseo; Dor local do enxerto.

\begin{abstract}
Objective: Compare the placement of absorbable jelly Gelfoan ${ }^{\circledR}$ versus the placement of a only a suction drain on the donor site, assessing post-operative pain, the presence of complications and the patient follow up. Methods: Through a computer program, 30 patients were randomized, of which 14 patients using Gelfoan ${ }^{\circledR}$ with an anesthetic and 16 patients using a suction drain. All patients were assessed in five consecutive periods, the pain was assessed based on the visual analogue scale (VAS), and the incidence of complications was observed. Patients with an anterior or posterior approach were included and only the iliac bone was considered the donor site. Patients between 18 and 70 years of age from both genders were included. Results: It was observed that the post-operative pain was less intense with the gelatin in the first assessments, but the mean results were similar and did not present statistical differences between both. In the average of the assessments, the visual analogue scale for pain was of two, not observing complications in the graft donor site. Conclusion: We have concluded that the approach with Gelfoan ${ }^{\circledR}$ with bupivacaine improves pain in the first 48 hours post-operatively when compared to the vacuum suction drain, but in average both techniques are safe, with the presence of residual pain in both interventions and a low complication rate, with none observed in our study.
\end{abstract}

Keywords: Prospective; Controlled; Born grafting; Donor site pain.

RESUMEN

Objetivo: Comparar la colocación de Gelfoan ${ }^{\circledR}$ con respecto a la colocación con solo el drenaje de succión en el área donante, evaluando el dolor posoperatorio, la presencia de complicaciones y el seguimiento de los pacientes. Metodología: Fueron randomizados mediante programa computacional 30 pacientes, de los cuales en 14 pacientes se utilizó Gelfoan ${ }^{\circledR}$ con anestésico y en 16 drenaje de succión. Todos los pacientes fueron evaluados en 5 periodos consecutivos. Fue evaluado el dolor basado en la Escala Visual Analógica (VAS), y observada la incidencia de complicaciones. Fueron incluidos pacientes con enfoque anterior y posterior, se consideró como fuente donadora única la cresta ilíaca. Se incluyeron pacientes de 18 a 70 años de ambos sexos. Resultados: se observó que el dolor posoperatorio disminuyó con gel en las primeras evaluaciones. Sin embargo, en media, el resultado fue similar, sin presencia de diferencia estadística entre ambas técnicas. Realizándose la media en las evaluaciones, la escala visual analógica de dolor fue de 2; no se observaron complicaciones en el área de extracción del injerto. Conclusiones: El abordaje con Gelfoan ${ }^{\circledR}$ con bupivacaina mejora el dolor en las primeras 48 horas poscirurgía, comparándolo con el drenaje de succión al vacío. Sin embargo, en media, ambas técnicas son seguras, presentándose dolor residual en las dos intervenciones con bajo índice de complicaciones. No se observaron complicaciones en el estudio realizado.

Palabras claves: Prospectivo, Controlado, Injerto óseo, Dolor local de injerto.

1. Medico Assistente do Serviço de Ortopedia eTraumatologia. Grupo de Patologias da Coluna Vertebral do Hospital Santa Marcelina, São Paulo, SP, Brasil.

2. Medico Assistente do Serviço de Ortopedia eTraumatologia. Chefe do Grupo de Patologias da Coluna Vertebral do Hospital Santa Marcelina, São Paulo, SP, Brasil.

3. Chefe do Serviço de Ortopedia eTraumatologia. Hospital Santa Marcelina, São Paulo, SP, Brasil.

Correspondência: Rua Santa Marcelina 177 Departamento de Ortopedia eTraumatologia, São Paulo, SP, Brasil. 08270-070. luizclr@terra.com.br

Trabalho realizado no Serviço de Ortopedia eTraumatologia. Grupo de Patologias da Coluna Vertebral do Hospital Santa Marcelina, São Paulo, SP, Brasil. 


\section{INTRODUÇÃO}

Células podem sobreviver por períodos diferentes. Em condições favoráveis elas podem manter sua viabilidade por um longo período apos serem retiradas do tecido de origem. Fáscia, osso e gordura são os tecidos mais favoráveis para doação. O menos favorável é o musculo ${ }^{1}$. A historia do uso dos enxertos teve início em botânica e é comumente realizado entre as plantas. Posteriormente iniciou-se o seu uso entre animais, onde os primeiros princípios observados eram estabelecer um suprimento nutricional rápido, uma boa área de contato e suporte mecânico ${ }^{1}$.

Nos seres humanos a primeira descrição do uso de enxerto ósseo data de 1668, quando um cirurgião alemão usou osso de cão para cobrir o crânio de um soldado, apresentando bom resultado. Devido à técnica o soldado foi excomungado e o médico só pode retornar para a igreja após remover o osso do paciente? ${ }^{2}$.

Atualmente o enxerto ósseo tem três funções fisiológicas sendo elas: osteoindução, que a condição da estimular a formação óssea atraindo células com condição para formar osso; osteocondução que é a condição de fornecer uma estrutura para a deposição óssea e por ultimo a osteogênese que é a formação óssea a partir de sua própria reserva celular ${ }^{3-5}$.

Na cirurgia da coluna vertebral a utilização de enxerto ósseo é uma rotina, porém a dor na região do ilíaco está presente entre três a $61 \%$ dos procedimentos ${ }^{6}$, além de outras complicações. Existem atualmente várias técnicas e diferentes procedimentos descritos ${ }^{7,8}$. Até este momento não existe um tratamento definido como padrão.

Este estudo tem por objetivo comparar duas formas de tratamento do sitio doador de enxertos ósseos, avaliando a colocação de gelatina absorvível (Gelfoan $\AA$ ) embebida em bupivacaina a $5 \%$ com vaso contrictor e o uso de um dreno de sucção a vácuo.

\section{MATERIAL E MÉTODO}

Foram randomizados 30 pacientes que após assinarem o Termo de consentimento livre esclarecido (TCLE), foram alocados em dois grupos. A randomização foi efetuada por programa de computador e colocados em envelopes opacos. Cada envelope era aberto no centro cirúrgico no momento do fechamento da região doadora no ilíaco.

Após a abertura do envelope o método indicado era utilizado. A avaliação utilizada foi a escala visual analógica (EVA) sendo cada avaliação feita por um avaliador independente que colhia os dados 12h, 24h 48h e com 15 dias e 30 dias de pós-operatório. Também foi incluída como critério de avaliação a presença de complicações locais, sendo avaliada a presença de hérnia muscular, infecção e parestesia do nervo cutâneo lateral da coxa.

Os critérios de inclusão foram pacientes de ambos os sexos que aceitaram participar do trabalho. Pacientes indicados para a realização que fossem submetidos à artrodese de coluna vertebral tanto anterior quanto posterior, sendo incluídas cirurgias degenerativas ou fraturas da coluna.

Critérios de exclusão foram pacientes com idade menor de 18 anos, pacientes que foram submetidos à cirurgia de escoliose e cirurgias tumorais, ou pacientes com capacidade cognitiva que não pudessem responder ao questionário.

Os pacientes, após randomização foram submetidos a um dos procedimentos, colocação de uma geléia absorvível (Gelfoan ${ }^{\circledR}$ ) embebida em bupivacaina com vaso constritor, outro grupo de indivíduos utilizaram somente dreno de sucção a vácuo. Devido a marcaina ser contra-indicada em pacientes abaixo de 12 anos, foram excluídas as cirurgias de deformidade e ampliando a margem de segurança, foram excluídos também pacientes menores de 18 anos, após a escolha da técnica. A sutura da fáscia foi feita de forma contínua com fio absorvível, sendo também fechado os planos superficiais. A pele foi suturada com fio absorvível tipo Monocril ${ }^{\circledR}$ com ponto intradérmico continuo. Foram incluídos pacientes com cirurgia anterior e posterior a técnica utilizada foi a mesma.

A retirada de enxerto foi sempre realizada por um cirurgião sênior, com pelo menos 5 anos de experiência em cirurgia da coluna vertebral.

A técnica da retirada do enxerto foi a mesma em todos os ca- sos, o enxerto foi retirado em tiras de $1 \mathrm{~cm}$ de largura por $4 \mathrm{~cm}$ de comprimento, sendo tirada de 3 a 4 tiras, após a retirada, foi feito a curetagem do osso esponjoso com uma cureta angulada. A cortical medial foi preservada em todos os casos, para evitar a invasão da cavidade abdominal. Na retirada por via posterior foi mantida uma distancia segura de $8 \mathrm{~cm}$ da espinha ilíaca póstero superior para evitar uma invasão da região da sacrilíacas. Na via anterior foi mantida uma distância de 2 a 3cm da espinha ilíaca anterossuperior.

\section{RESULTADOS}

Foram incluídos 30 pacientes sendo 16 do sexo masculino e 14 do sexo feminino; a idade media no momento da abordagem foi de 45,5 anos (Tabela 1) quanto ao tipo de cirurgia foram 21 abordagens por via posterior e nove abordagens por via anterior. Quanto a forma de tratamento 14 pacientes utilizaram Gelfoan $₫$ com bupivacaina e 16 pacientes utilizaram dreno de sucção (Tabela 2). Como não foi observada nenhuma complicação em nenhum dos pacientes esta avaliação não foi incluída na analise estatística.

\section{Comparação entre as diferentes variáveis}

Não foram encontradas diferenças significativas entre os valores de idade dos pacientes na cirurgia segundo o gênero (teste Mann-Whitney com $p=0,328$ ) (Tabela 3). Quando foram comparados a abordagem com o gênero também não encontramos diferenças significativas entre estas proporções (teste exato de Fisher com $\mathrm{p}=0,440$ ).

Não encontramos diferenças significativas quando comparamos a diferentes abordagem e o gênero (teste qui-quadrado com $p=0,282)$. Os dados referentes à idade apresentaram distribuição normal (teste Kolmogorov-Smirnov). Observamos que a média de Idade para a abordagem anterior é significativamente maior que a da abordagem posterior (teste $t$ Student com $p=0,047$ ). Quando analisamos os dados referentes à idade as mesmas apresentaram distribuição normal (teste Kolmogorov Smirnov). Não existe diferença significativa entre as médias das idades dos pacientes segundo as formas de tratamento de enxerto (teste $t$ Student com $p=0,993$ ). Quando avaliamos o tipo de cirurgia não encontramos diferenças significativas entre as diferentes proporções da abordagem cirúrgica e a forma de tratamento do enxerto (teste exato de Fisher com $p=1,000$ ) (Tabela 4). Na avaliação da EVA, quando comparamos o resultado em relação ao tipo de abordagem cirúrgica observamos que não existem diferenças significativas entre os valores da média dos pacientes da escala visual analógica de dor (EVA) segundo o tipo de cirurgia (teste Mann Whitney com $p=0,759$ ) (Figura 1).

$\mathrm{Na}$ primeira avaliação, encontramos que os valores da escala visual analógica nas 12 primeiras horas para o grupo que utilizou o dreno são significativamente maiores que o grupo Gelfoan ${ }^{\circledR}$ (teste Mann Whitney com $p=0,028$ ). Avaliação com 24h, os valores da escala visual de dor EVA para o grupo dreno de sucção são signi-

Tabela 1. Média de idade dos pacientes.

\begin{tabular}{c|c|c|c}
\hline \multirow{4}{*}{ idade } & \multicolumn{2}{|c|}{ Média } & 47,2 \\
\cline { 2 - 3 } $\begin{array}{c}\text { 95\% intervalo de confiança } \\
\text { Media }\end{array}$ & Limite inferior & 43,2 \\
\cline { 2 - 3 } & \multicolumn{2}{|c|}{ Limite superior } & 51,2 \\
\cline { 2 - 3 } & \multicolumn{2}{|c|}{ Mediana } & 45,5 \\
\cline { 2 - 3 } & \multicolumn{2}{|c|}{ Desvio Padrão } & 10,7 \\
\cline { 2 - 3 } & \multicolumn{2}{c}{ Mínimo } & 31,0 \\
\cline { 2 - 3 } & \multicolumn{2}{c}{ Máximo } & 67,0 \\
\hline
\end{tabular}

Tabela 2. Relação do sexo com o tipo de abordagem da coluna.

\begin{tabular}{c|c|c|c|c|c}
\hline & & & \multicolumn{2}{|c|}{ Tipo de Cirurgia } & \multirow{2}{*}{ Total } \\
\hline & & & Anterior & Posterior & \\
\hline \multirow{3}{*}{ sexo } & \multirow{2}{*}{ Masculino } & indivíduos & 6 & 10 & 16 \\
\cline { 3 - 6 } & \multirow{2}{*}{ Total } & $\%$ SEXO & $37,5 \%$ & $62,5 \%$ & $100,0 \%$ \\
\cline { 3 - 6 } & \multirow{2}{*}{ Feminino } & indivíduos & 3 & 11 & 14 \\
\cline { 3 - 6 } & $\%$ SEXO & $21,4 \%$ & $78,6 \%$ & $100,0 \%$ \\
\hline & & indivíduos & 9 & 21 & 30 \\
\hline
\end{tabular}


Tabela 3. Relação entre a media de idade e o gênero dos pacientes.

\begin{tabular}{|c|c|c|c|c|}
\hline & \multicolumn{3}{|c|}{ Sexo } & \multirow{3}{*}{$\begin{array}{c}\text { Estatística } \\
49,4 \\
43,4\end{array}$} \\
\hline \multirow{14}{*}{ Idade na cirurgia } & \multirow{7}{*}{ Masculino } & \multicolumn{2}{|c|}{ Média } & \\
\hline & & $95 \%$ intervalo & $\begin{array}{l}\text { Limite } \\
\text { inferior }\end{array}$ & \\
\hline & & $\begin{array}{l}\text { de contlança } \\
\text { para a média }\end{array}$ & $\begin{array}{l}\text { Limite } \\
\text { superior }\end{array}$ & 55,4 \\
\hline & & med & & 46,0 \\
\hline & & Desvio & & 11,3 \\
\hline & & Mín & & 34,0 \\
\hline & & Máx & & 67,0 \\
\hline & \multirow{7}{*}{ Feminino } & \multicolumn{2}{|c|}{ Media } & 44,8 \\
\hline & & \multirow{2}{*}{$\begin{array}{l}95 \% \text { Intervalo } \\
\text { de confiança } \\
\text { para a média }\end{array}$} & $\begin{array}{l}\text { Limite } \\
\text { inferior }\end{array}$ & 39,1 \\
\hline & & & $\begin{array}{c}\text { Limite } \\
\text { superior }\end{array}$ & 50,5 \\
\hline & & \multicolumn{2}{|c|}{ Mediana } & 45,5 \\
\hline & & \multicolumn{2}{|c|}{ Desvio padrão } & 9,9 \\
\hline & & \multirow{2}{*}{\multicolumn{2}{|c|}{ Mínimo }} & 31,0 \\
\hline & & & Máximo & 60,0 \\
\hline
\end{tabular}

Tabela 4. Tipo de cirurgia $x$ forma de tratamento no enxerto.

\begin{tabular}{c|c|c|c|c|c}
\hline & & & \multicolumn{2}{|c|}{ Tratamento } & \multirow{2}{*}{ Total } \\
\hline \multirow{3}{*}{ Cirurgia } & \multirow{2}{*}{ Anterior } & Indivíduos & 4 & 5 & 9 \\
\cline { 3 - 5 } & \multirow{2}{*}{ Total } & Relação intergrupo & $44,4 \%$ & $55,6 \%$ & $100,0 \%$ \\
\hline & & Indivíduos & 10 & 11 & 21 \\
\cline { 3 - 6 } & & Relação intergrupo & $47,6 \%$ & $52,4 \%$ & $100,0 \%$ \\
\hline \multirow{2}{*}{ Indivíduos } & 14 & 16 & 30 \\
\hline & & $\begin{array}{c}\text { Relação total } \\
\text { entre os grupos. }\end{array}$ & $46,7 \%$ & $53,3 \%$ & $100,0 \%$ \\
\hline
\end{tabular}

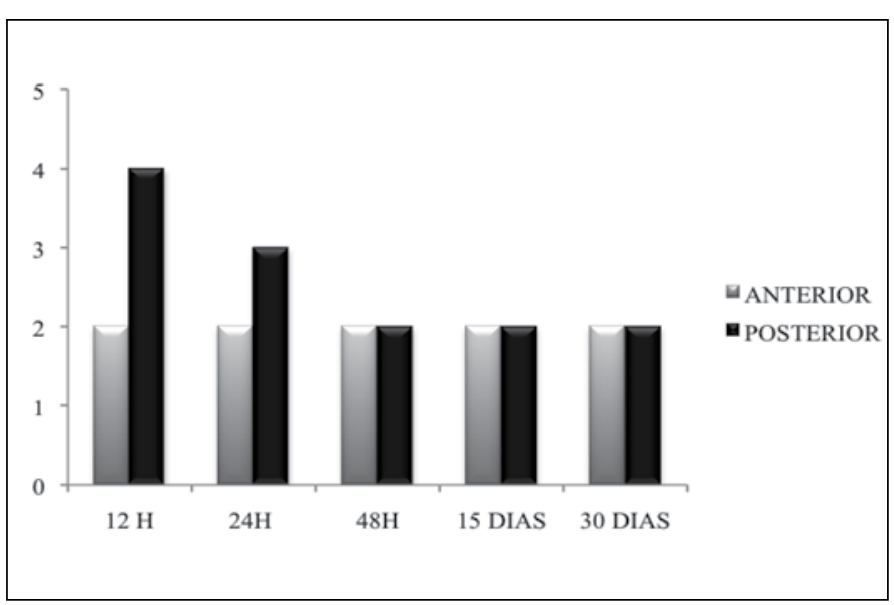

Figura 1. Relação entre a variável dor e tipo de abordagem cirúrgica.

ficativamente maiores que o grupo Gelfoan ${ }^{\circledR}$ (teste Mann Whitney com $p=0,003)$.

Na terceira avaliação não encontramos diferenças significativas entre os valores da escala visual de dor EVA nas 48 primeiras horas segundo a forma de tratamento dos enxertos (teste Mann Whitney $\operatorname{com} p=0,223)$.

Nas avaliações subsequentes após a alta, os valores da escala visual no primeiro retorno para o grupo dreno são significativamente maiores que para o grupo Gelfoan ${ }^{\circledR}$ (teste Mann Whitney com $p=0,003)$ e também não existem diferenças significativas entre os valores da escala visual no segundo retorno segundo a forma de tratamento dos enxertos (teste Mann Whitney com $p=0,126$ ).

Foi realizada uma avaliação, baseada na media das analises onde observamos que não existem diferenças significativas entre os valores da escala visual de dor EVA média segundo a forma de tratamento dos enxertos (teste Mann Whitney com $\mathrm{p}=0,241$ ) (Figura 2).

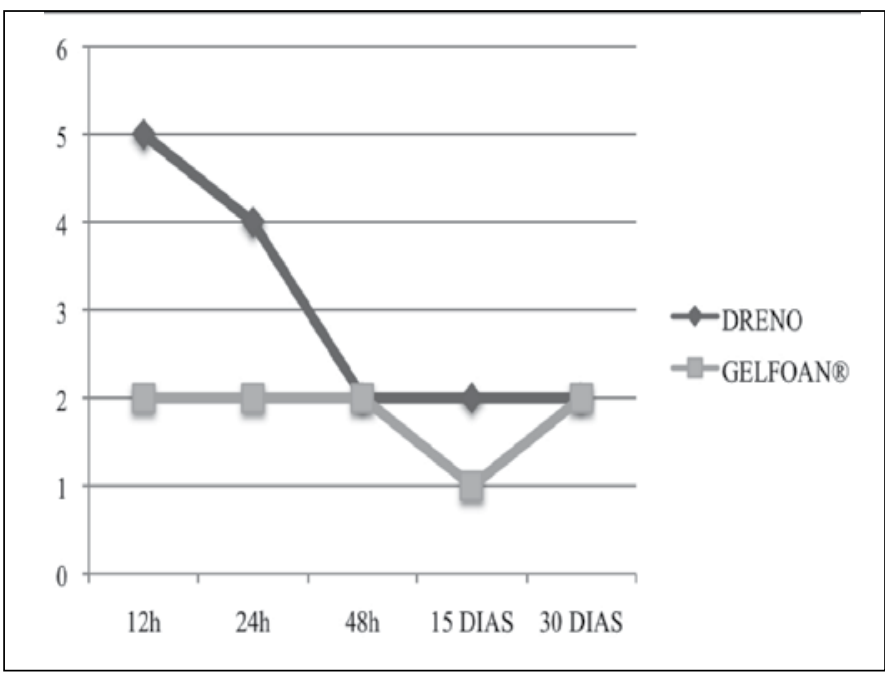

Figura 2. Evolução da dor durante a evolução nos diferentes tipos de tratamento.

\section{DISCUSSÃO}

A abordagem para retirada do enxerto baseada em critérios anatômicos é segura. Quando respeitamos as referências para evitar a invasão da sacro-ilíacas ou anterior com margem de segurança da espinha ilíaca anterossuperior, a presença de complicações pode ser evitada. Alguns trabalhos sugerem uma nova abordagem como Hutchinson et al. ${ }^{9}$ onde a abordagem se faz pela linha média. Nas cirurgias da coluna vertebral a principal abordagem é na região lombo-sacro, este tipo não pode ser realizada, mas em artrodeses mais altas tal técnica é factível.

Lawrence et al. ${ }^{10} \mathrm{em}$ uma revisão do sitio do enxerto observaram inúmeras complicações, entre elas; lesão nervosa e arterial, deformidades cosméticas e infecç̃os, fatos estes não observados nos indivíduos do presente estudo. Porém ele observa que a dor pode ser encontrada em $15 \%$ dos pacientes, em nosso estudo a dor, de forma estatística, esteve presente em todos os pacientes.

Charles et al. ${ }^{11}$ fez um estudo com colágeno microfibrilar onde a pesquisa investigava a hemostasia, em nosso trabalho não tivemos intercorrência hemorrágicas, apesar de o volume drenado não ter sido um parâmetro de avaliação de forma estatística, em nenhum deles foi observado um volume acima de $200 \mathrm{ml}$. Não observamos a presença de coleções hemáticas nos pacientes com uso de gelatina absorvível, onde foi observado apenas pequeno hematoma ao redor da ferida em ambos os pacientes.

Ao contrario de Burtein et al ${ }^{12}$ comparamos duas formas de tratamento do sitio doador, para que o trabalho fosse o mais coerente possível padronizamos a técnica para retirada do tecido ósseo do ilíaco, aquele autor comparava técnica aberta com técnica minimamente invasiva e o mesmo observou que a abordagem minimamente invasiva apresentava menor índice de complicações; como nossa abordagem se mostrou segura e forneceu uma quantidade óssea suficiente, para a cirurgia da coluna vertebral a possibilidade de mínima abordagem com necessidade de uma maior quantidade de enxerto pode aumentar a chance de invasão da cavidade abdominal ou outras complicações, principalmente em pacientes obesos, onde os parâmetros podem ser mais imprecisos.

Nosso estudo foi prospectivo e controlado e concordamos com Banwart et al..$^{13}$ que relata que complicações maiores são incomuns, mas que complicações menores estão presentes. Nosso estudo evidenciou apenas dor como complicação, o autor citado relata que uma sutura bem realizada e sem tensão melhora o resultado pós-cirúrgico. Realizamos a sutura de forma cautelosa após hemostasia rigorosa e uma aproximação dos bordos de forma regular e homogênea. 
Christopher et al. ${ }^{14}$ sugerem que a fonte doadora de enxerto deve ser tratada com Gelfoan ${ }^{\circledR}$ e dreno de sucção, detalhe este não comprovado em nosso trabalho onde avaliamos que o dreno de sucção isolado é seguro, e o uso de gelatina com anestésico também; não necessitando da utilização das duas técnicas. Roidis et al..$^{15}$ apresentaram resultados onde apontam que a abordagem posterior foi mais segura que a anterior. Nossos resultados não mostram diferença entre as diferentes abordagens. A análise da dor em relação a via utilizada foi semelhante.

\section{CONCLUSÃO}

O uso de Gelfoan ${ }^{\circledR}$ com anestésico melhora a dor no pós-operatório imediato, assim como nas primeiras 24 horas, mas na média as duas formas de tratamento são semelhantes. A incidência de complicações é baixa em ambas a situações.

A incidência de dor após a retirada do enxerto, na abordagem posterior ou anterior foi semelhante. A colocação de Gelfoan ${ }^{\circledR}$ ou dreno são factíveis, não influenciando na evolução do quadro álgico. O respeito a critérios anatômicos bem definidos na retirada do enxerto garante um procedimento seguro e com baixo índice de complicações.

\section{REFERÊNCIAS}

1. Abee FH. The Fundamental Principles Involved in the use of the Bone Graft in Surgery. The American Journal of the Medical Sciences (Philadelphia)149(3) 313-25,1915.

2. Meekeren J. Van. Heel en Geneeskonstige Aanmerkingen. Amsterdan, commelijn, 1668.

3. Bieber EJ.; Wood MB. Bone Recosntruction. Clin Plast Surg 13:645. 1986.

4. Burchardt H.:The Biology of Bone Graft Repair. Clin Orthop.174:28 1983.

5. Lacroix P.Organizers and the Growth of Bone: J Bone and Joint Surg. 29 A 292; 1947.

6. Acharya NK, Mahajan CV, Kumar RJ, Varma HK, Menon VK. Can iliac crest reconstruction reduce donor site morbidity? a study using degradable hydroxyapatite-bioactive glass ceramic composite. J Spinal Disord Tech. 2010:23(4):266-71.

7. Colterjohn NR, Bednar DA. Procurement of bone graft from the iliac crest. An operative approach with decreased morbidity. J Bone Joint Surg Am. 1997;79:756-9.

8. Banwart JC, Asher MA, Hassanein RS. lliac crest bone graft harvest donor site morbidity: a statistical evaluation. Spine. 1995;20:1055-60.

9. Hutchinson MR, Dall BE. Midline fascial splitting approach to the iliac crest for bone graft.
A new approach. Spine (Phila Pa 1976). 1994:19(1):62-6.

10. Kurz LT, Garfin SR, Booth RE. Harvesting autogenous iliac bone grafts. A review of complications and techniques. Spine (Phila Pa 1976). 1989;14(12):1324-31.

11. Craig CC. Asher MA.Hemostasis in Human Iliac Crest Donor Sites With Microfibrilar Colagen. Spine 1977;(2)313-7.

12. Burstein FD. Simms C. Cohen SR. Work F. Pachal M. lliac Crest Bone Grafy Harvesting Techiniques: A Comparison. Plastic and Reconstructive Surgery. 2000;34-9.

13. Banwart JC, Asher MA, Hassanein RS. lliac crest bone graft harvest donor site morbidity. A statistical evaluation. Spine (Phila Pa 1976). 1995:20(9):1055-60

14. Finkemeier CG. Bone-grafting and bone-graft substitutes. J Bone Joint Surg Am. 2002;84$A(3): 454-64$

15. Ahlmann E, Patzakis M, Roidis N, Shepherd L, Holtom P. Comparison of anterior and posterior iliac crest bone grafts in terms of harvest-site morbidity and functional outcomes. J Bone Joint Surg Am. 2002;84-A(5):716-20. 